\title{
Percutaneous Adhesiolysis Versus Transforaminal Epidural Steroid Injection for the Treatment of Chronic Radicular Pain Caused by Lumbar Foraminal Spinal Stenosis: A Retrospective Comparative Study
}

\author{
Yongbum Park, MD' ${ }^{1}$, Woo Yong Lee, $\mathrm{MD}^{2}$, Jae Ki Ahn, $\mathrm{MD}^{1}$, Hee-Seung Nam, MD ${ }^{3}$, Ki Hoon Lee, $\mathrm{MD}^{1}$
}

Departments of ${ }^{1}$ Rehabilitation Medicine and ${ }^{2}$ Anesthesiology, Sanggye Paik Hospital, Inje University College of Medicine, Seoul; ${ }^{3}$ Yonsei S Rehabilitation Clinic, Seoul, Korea

\begin{abstract}
Objective To investigate the efficacy of percutaneous adhesiolysis (PA) compared to fluoroscopy (FL)-guided transforaminal epidural steroid injection (TFESI) in patients with radicular pain caused by lumbar foraminal spinal stenosis (LFSS) by assessing pain relief and functional improvement at 4 and 12 weeks post-procedure.

Methods This retrospective study included 45 patients who underwent PA or FL-guided TFSEI for radicular pain caused by LFSS of at least 3 months' duration. Outcomes were assessed with the Oswestry Disability Index (ODI) and Verbal Numeric Pain Scale (VNS) before the procedure and at 4 and 12 weeks post-procedure. A successful outcome was defined by $>50 \%$ improvement in the VNS score and $>40 \%$ improvement in the ODI score.

Results ODI and VNS scores improved 4 and 12 weeks post-procedure in both groups. Statistically significant differences between groups were observed in ODI and VNS at 12 weeks $(\mathrm{p}<0.05)$. The proportion of patients with successful outcomes was significantly different between the two groups only at the 12-week time point.

Conclusion Our study suggests that PA is effective for pain reduction and functional improvement in patients with chronic radicular pain caused by LFSS. Therefore, PA can be considered for patients with previous ineffective responses to conservative treatment. Although PA seems to be more effective than TFEFI according to the results of our study, in order to fully elucidate the difference in effectiveness, a prospective study with a larger sample size is necessary.
\end{abstract}

Keywords Adhesiolysis, Fluoroscopy, Epidural injection 


\section{INTRODUCTION}

Lumbar foraminal spinal stenosis (LFSS) is a common cause of radicular pain [1]. An $8 \%$ to $11 \%$ incidence of lateral root entrapment has been reported [1-3]. Although few studies have examined the mechanical compression of nerve roots in LFSS, it has been hypothesized that continuous compression of nerve roots damages microvessels and leads to ischemia, edema, demyelination, and C-fiber activation [4]. Various types of conservative treatments have been used for patients with radicular pain caused by LFSS. Fluoroscopy (FL)-guided transforaminal epidural steroid injection (TFESI) is considered the most precise and effective route of steroid administration $[5,6]$. The transforaminal approach is advantageous because corticosteroid preparations can be injected close to the probable source of the irritated nerve root, and this approach results in better ventral epidural spreading than does the interlaminar approach $[5,6]$.

Although previous studies demonstrate efficacy of TFESI in managing lumbar radicular pain [6-8], epidural steroid injection is occasionally not effective in managing leg pain [8-10]. It is thought that epidural fibrosis leads to imprecise drug delivery in some cases, which limits the effectiveness of TFSEI. Furthermore, epidural fibrosis effectively tethers the dura and nerve roots, causing a significant subset of patients to experience chronic radicular pain [11-13]. The percutaneous adhesiolysis (PA) technique could overcome the limitations of TFESI. A PA catheter enters the ventral epidural space directly, advancing along the ventral epidural space (lateral recess to foramen) and directly breaking up perineural/epidural adhesions, fibrosis, or other resistive areas that are physical barriers to penetration of perineurally deposited drugs; this may enhance the delivery of high concentrations of injected drugs to the target area [11-13]. A small number of studies have addressed the effectiveness of PA in patients with radicular pain caused by LFSS; also, only a few studies have compared the effectiveness and safety of PA and TFESI. Hence, the purpose of this retrospective study was to assess the relative effectiveness of PA and TFESI for managing radicular pain in patients with LFSS.

\section{MATERIALS AND METHODS}

\section{Study design}

We retrospectively compared data from patient charts. Patient privacy and data confidentiality were maintained throughout the research process. Approval from the Institutional Review Board of the Sanggye Paik Hospital was obtained. The approval included a waiver of informed consent, since the study did not include direct contact with the study population and all patient identifiers were removed from the data set upon initial collection.

\section{Subjects}

We studied data from patients with lumbar radicular pain caused by LFSS who visited our pain clinic between June 1, 2013 and June 31, 2014. Diagnoses were based on clinical profiles, physical examinations, electromyography tests, and computed tomography (CT) or magnetic resonance imaging (MRI) scans [14].

Those who met the following inclusion criteria were selected: aged 18 or older, received PA or FL-guided TFESI, no previous lumbar surgery, no central canal stenosis, no lateral recess stenosis no spondylolisthesis evident on MRI or CT, absence of progressive motor deficit or significant sensory deficit, and absence of cauda equina syndrome. We only included patients who had experienced chronic radicular pain for at least 3 months and had failed to respond to anti-inflammatory medications, analgesic therapy, or physical therapy for at least 4 weeks. Exclusion criteria included a diagnosis of sacroiliac joint or facet joint pain based on clinical or radiological evaluation, presence of definite motor weakness or hypoesthesia, presence of psychiatric disorders, and laboratory results suggesting bleeding disorders, infection, inflammatory disease, or rheumatoid disorders.

\section{Percutaneous adhesiolysis}

PA and FL-guided TFESI were both commonly used in our clinic to treat lumbar radicular pain caused by LFSS. Patients who failed to respond to more conservative management were informed of the potential risks associated with the two procedures and the benefits and risks of using corticosteroid mixed with contrast media, then asked to provide consent. The choice between PA and FL was made by the patient.

FL-guided TFESI was conducted by a physician (Y. 
Park) with $>7$ years of experience and PA was performed by a pain specialist (W. Y. Lee) with $>3$ years of experience. FL-guided TFESI was performed as an outpatient procedure and PA was performed in an operating room.

Before the PA procedure, an intravenous catheter was placed and antibiotics were administered. PA was performed using an EpiStim catheter (Sewoon Medical, Seoul, Korea) under sterile conditions using FL with continuous monitoring of vital signs (blood pressure, pulse, and heart rate). With the patient in the prone position, the site of needle insertion was sterilized with povidone iodine and draped. The FL was adjusted over the lumbosacral area such that a caudal approach could be used in both the anteroposterior and lateral views. After appropriate positioning of the FL, the precise needle insertion area around the sacral hiatus was determined and infiltrated with local anesthetics. A 15-gauge RK needle (Sewoon Medical) was inserted into the epidural space and a 19-gauge EpiStim catheter was advanced through the needle up to the 3rd sacral vertebra. An epidurogram was obtained after injecting approximately $2-5 \mathrm{~mL}$ of contrast media to confirm that the needle was placed in the epidural space and to avoid intravascular or subarachnoid needle placement. The tip of the catheter was positioned to address the adhesion site as identified by filling defects and suspected pain source. Subsequently, adhesiolysis and decompression were carried out by distension with a mixture $10 \mathrm{~mL}$ of $0.25 \%$ bupivacaine and $3 \%$ hypertonic saline, and by mechanical means using the catheter. When the catheter was placed in an area suspected to be the source of pain, some patients indicated that they felt pain similar to what they had been experiencing. After adhesiolysis, sufficient filling of the target nerve roots and epidural space was confirmed without intravascular, subarachnoid, or extra epidural injections. Then, a mixture of $5 \mathrm{~mL} 0.25 \%$ bupivacaine, $2 \mathrm{mg}$ betamethasone, and 1,500 units of hyaluronidase was slowly injected (Fig. 1A). After completion of the procedure, the patient was placed in a supine position and transferred to the recovery room. In the recovery room, the patient was closely monitored for any potential complications or adverse effects before being discharged.

\section{Fluoroscopy-guided transforaminal epidural steroid injection}

All patients were placed in a prone position with a pillow under the lower abdomen and above the iliac crest to reduce lumbar lordosis. The FL was adjusted so that an $\mathrm{X}$-ray passed perpendicular to the endplate of the lower portion of the upper vertebrae, which is one of the two targeted spinal segments. After confirming overlap between the pedicle of the upper vertebrae and the superior
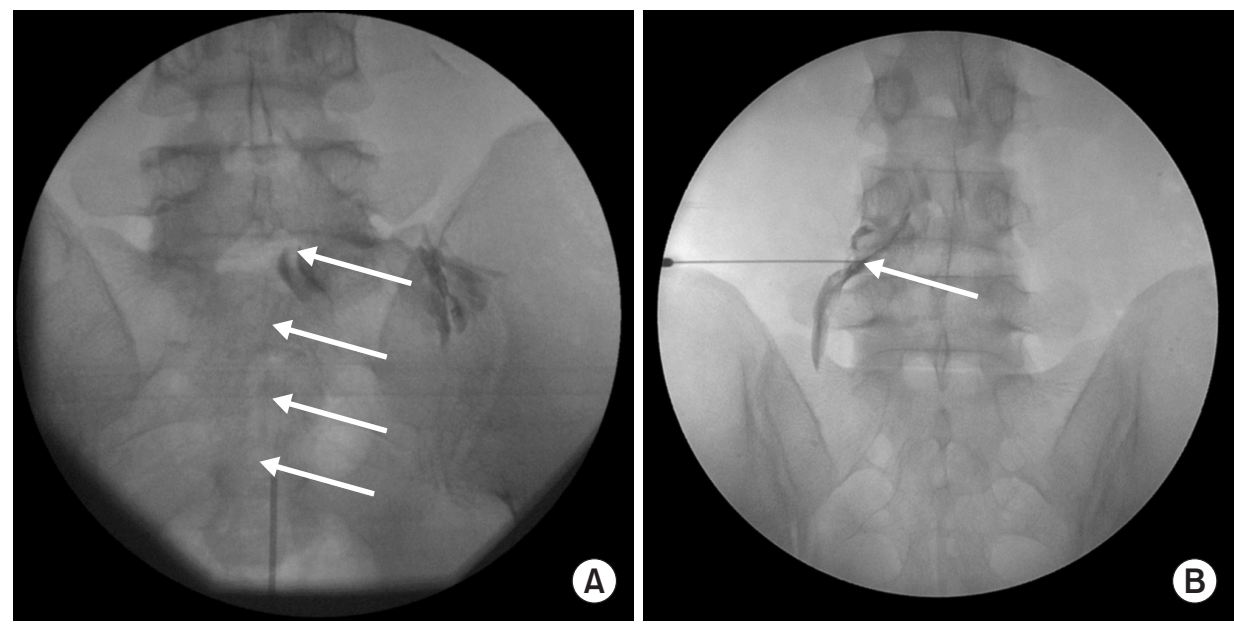

Fig. 1. (A) Percutaneous adhesiolysis approach to the L5 nerve root. The anterior-posterior view shows the proper location of catheter in the L5-S1 foraminal canal (arrow). After adhesiolysis, sufficient filling of the target nerve roots and epidural space was confirmed without intravascular, subarachnoid, or extra epidural injections. Conventional transforaminal approach to the L5 nerve root. (B) Conventional transforaminal approach to the L4 nerve root. The anteriorposterior view shows the proper location of the needle at the base of the pedicle (arrow). A small amount of contrast media was used to confirm epidural spread. 
articular process of the lower vertebrae from an oblique view, a spot directly inferior to the pedicle was identified as the target. The area was prepared for injection by applying an aseptic dressing after sterilizing the skin with betadine and alcohol. The tip of a 22-gauge, 3.5-inch spinal needle was gradually advanced from the 6 o'clock direction toward the spot directly inferior to the pedicle using radiologic guidance. Although the needle tip did not have to proceed all the way to the periosteum bone, we ensured that the tip was placed directly inferior to the pedicle and in the inferolateral area of the intra-articular space (i.e., safe triangle) via several radiologic views. When the tip touched the inferolateral border, the FL was turned to the lateral view, and the needle was advanced gradually toward the foramen in an anterior and superior direction. Drug delivery into the anterior epidural space was confirmed via injection of 1-2 mL radio contrast media under intermittent fluoroscopic imaging (Fig. 1B). If it was not observed, $2 \mathrm{~mL}$ of the drug $(0.5 \%$ lidocaine $1.5 \mathrm{~mL}+$ triamcinolone $20 \mathrm{mg}$ ) was injected. We defined failure of TFESI as a $50 \%$ or less reduction on the Verbal Numeric Pain Scale (VNS) compared to pre-injection scores, when at least 2 injections were administered over 3 months.

\section{Review of clinical data}

Ninety-two patients (PA, n=28; FL-TFESI, n=64) received treatment during the time period encompassed by this study, 78 of whom (85\%) met the inclusion criteria. Twenty-two (24\%) patients were excluded because the patient did not complete and return the follow-up survey. Eleven (12\%) patients were excluded because of the exclusion criteria listed above. Finally, 15 patients treated with PA and 30 patients treated with FL-TFESI were left. A standardized chart abstraction form was used to extract the collected data on demographics, treatment, pain severity, and functional assessment. Follow-up interviews were performed by nursing personnel not involved in the original procedure. The VNS and ODI were used to evaluate clinical effectiveness in terms of pain reduction and functional improvement at 4 and 12 weeks after the last injection. On the VNS, a score of 0 indicates no pain, and a score of 10 indicates the worst pain imaginable [15]. The ODI is one of the most commonly used diseasespecific readouts for patients with LBP [16]. The ODI is consists of ten items, each of which is scored from 0 to 5 ; the total is added and multiplied by 2 to produce a final score in the range of $0-100$.

Successful outcome was defined as a reduction in the VNS score of $50 \%$ or more, and successful functional improvement was defined as a reduction in the ODI of $40 \%$ or more at 4 weeks and 12 weeks after the procedure [17]. If the outcomes were not successful at 4 weeks, the patients were excluded at 12 weeks evaluation.

We reviewed patient charts to check for immediate adverse events (within a few minutes after the injection). Each patient was handed a questionnaire at the end of the procedure, and asked to complete it within 48 hours and return it at the 2-week follow-up visit.

\section{Statistics}

The chi-square test, Fisher exact test, and Mann-Whitney $U$ test were used to compare the characteristics of the two groups in variables such as sex, age, body mass index (BMI), target nerve root, and pain duration. At each time point, VNS and ODI were compared by repeated measure analysis of variance (ANOVA), and Bonferroni correction was conducted for post-hoc comparison. The chi-square test was used to assess differences in proportions. Fisher exact test was used wherever the expected value was less than 5. Statistics were performed with SAS Enterprise Guide 4.1 (4.1.0.471); $\mathrm{p}<0.05$ was considered statistically

Table 1. General characteristics of the patients

\begin{tabular}{lccc}
\hline & $\begin{array}{c}\text { PA } \\
(\mathbf{n = 1 5})\end{array}$ & $\begin{array}{c}\text { FL-guided } \\
\text { TFESI }(\mathbf{n}=\mathbf{3 0})\end{array}$ & p-value \\
\hline Age $(\mathrm{yr})$ & $61.1 \pm 12.8$ & $57.7 \pm 10.1$ & 0.381 \\
Female & $11(73.3)$ & $24(80.0)$ & \\
Male & $4(26.7)$ & $6(20.0)$ & 0.612 \\
BMI $\left(\mathrm{kg} / \mathrm{m}^{2}\right)$ & $24.83 \pm 3.05$ & $22.77 \pm 4.32$ & 0.072 \\
Duration (mo) & $7.1 \pm 4.3$ & $8.2 \pm 1.8$ & 0.344 \\
Target root & & & \\
L3 & $1(6.7)$ & $2(6.7)$ & \\
L4 & $3(20.0)$ & $3(10.0)$ & \\
L5 & $10(66.7)$ & $23(76.7)$ & \\
S1 & $1(6.7)$ & $2(6.7)$ & 0.829 \\
\hline
\end{tabular}

Values are presented as mean \pm standard deviation or number (\%).

PA, percutaneous adhesiolysis; FL-guided TFESI, fluoroscopy-guided transforaminal epidural steroid injection; BMI, body mass index.

Statistically significant as $\mathrm{p}<0.05$. 
Table 2. Comparison of VNS and ODI scores from baseline to 4 and 12 weeks post-procedure

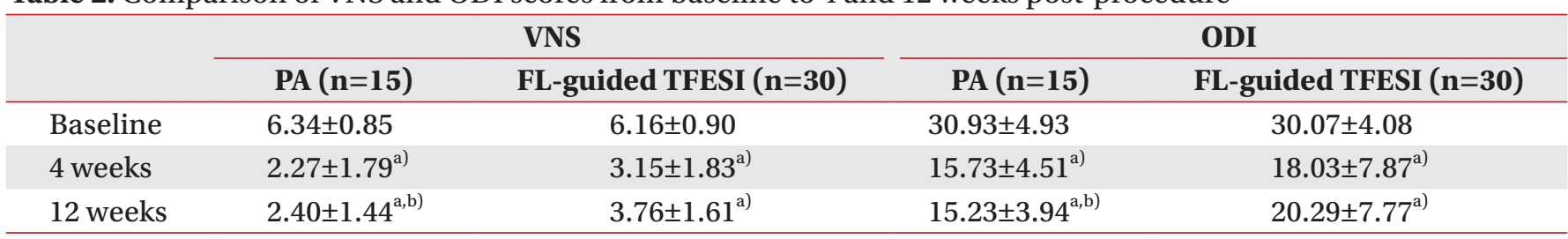

Values are presented as mean \pm standard deviation.

PA, percutaneous adhesiolysis; FL-guided TFESI, fluoroscopy-guided transforaminal epidural steroid injection; VNS, Verbal Numeric Pain Scale; ODI, Oswestry Disability Index.

${ }^{a)} \mathrm{p}<0.05$, significantly different before and after injection.

${ }^{b)} \mathrm{p}<0.05$, comparison of PA and FL-guided TFESI.

significant.

\section{RESULTS}

The average age of the patients was not significantly different between the two groups (PA, 61.1 \pm 12.8 years; FLTFESI, 57.7 \pm 10.1 years). Significant differences were not observed in general characteristics of such as sex, age, BMI, target nerve root, disease duration, and imaging results (Table 1).

The ODI and VNS showed significant improvements 4 and 12 weeks post-procedure in both groups. A significant between-group difference was observed in ODI and VNS scores at 12 weeks post-procedure (Table 2). The proportion of patients with $>50 \%$ improvement in VNS score and $>40 \%$ improvement in ODI score is illustrated in Fig. 2, which shows a $73.3 \%$ improvement in the PA group and a $43.3 \%$ improvement in the FL group at 12 weeks. Successful treatments intend to be different between the groups at 12 -week outcomes $(\mathrm{p}=0.057)$.

There were no cases of bent needle tips, torn catheters, sheared catheter remnants, or intrathecal placement of catheters. No instances of infection or hematoma were recorded. The only immediate adverse event reported was transient nerve irritation during the procedure, the incidence of which did not much differ between groups (FL-TFESI, $\mathrm{n}=4$; PA, $\mathrm{n}=3$ ).

\section{DISCUSSION}

The initial treatment of radicular pain associated with LFSS is conservative and includes mobilization exercises, anti-inflammatory medications, activity modifications, and epidural corticosteroid injections. TFESI may pro-

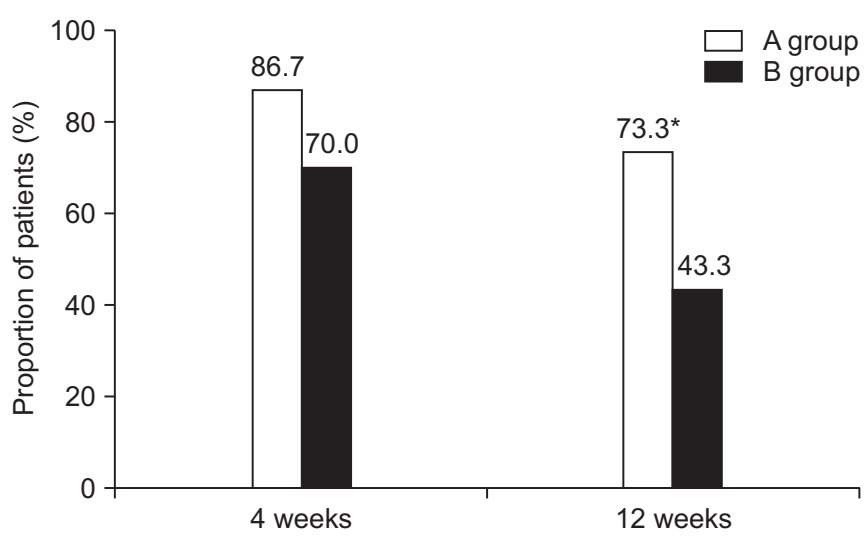

Fig. 2. Illustration of significant pain relief $(\geq 50 \%$ reduction in Verbal Numerical Pain Scale from baseline), functional improvement ( $\geq 40 \%$ improvement in the Oswestry Disability Index from baseline). A group is percutaneous adhesiolysis, B group is fluoroscopy-guided transforaminal epidural steroid. ${ }^{*} \mathrm{p}<0.05$, significant difference from baseline values.

vide pain relief and functional improvement in cases of central or lateral recess stenosis, but often does not alter the symptoms of foraminal stenosis [18]. Although surgery may be recommended for patients who do not respond to conservative treatments, older individuals with comorbidities are not always good surgical candidates. In LFSS, narrowing of the bony foramen causes mechanical compression of spinal nerve roots. This dynamic mechanical compression of the nerve root sheath manifests as neural hyperemia, venous congestion, and edema. Venous congestion has been suggested as the essential factor precipitating circulatory disturbance, thus inducing neurogenic claudication [19]. Perineural fibrosis is closely related to venous obstruction and may further impede nutrient transfer and predispose to nerve stretch 
injury [20]. Also, fibrosis may extend into the neural canal and adhere to the dura mater and nerve roots, causing adhesion of nerve roots or dura, which may in turn contribute to persistent radicular pain and prevent delivery of medications for pain relief [21].

PA removes barriers in the epidural space thought to contribute to painful processes and prevent delivery of pain-relieving drugs $[11,12,21]$. PA may also wash out inflammatory cytokines from the affected area [22]. A systemic review examined the relationship between the volume of fluid introduced during epidural corticosteroid injection and relief of radicular pain [23]. The study found that larger injection volume was associated with a greater magnitude of pain relief. The proposed mechanisms through which additional volume contributes to pain relief include not only adhesiolysis and washout of inflammatory cytokines, but also lavage of the epidural space, suppression of ectopic discharge from injured nerves, and enhanced blood flow to ischemic nerve roots $[22,23]$. Previous studies investigated the efficacy of PA in lumbar spinal stenosis (LSS) [24-26]. Manchikanti et al. [24] reported that $76 \%$ of patients who underwent PA obtained significant pain relief at a 12-month follow-up, whereas only $12 \%$ of those with caudal block obtained pain relief. Another study by Manchikanti et al. [25] showed that $89 \%$ of patients with moderate or severe LSS had significant pain relief at 3 months, but in that study they performed repetitive procedures. In a different retrospective study, of 63 patients who were followed up at 3 months, 34 (54.0\%), 32 (50.8\%), and 30 (47.6\%) patients showed successful results on Numeric Rating Scale for back pain, Numeric Rating Scale for leg pain, and the ODI scale at 3 months, respectively [26].

In both studies by Manchikanti et al. [25,26], repetitive procedures were done and foraminal stenosis patients were excluded; also, they one of those studies did not compare the effectiveness of PA to that of TFESI. To our knowledge, this is the first study comparing the effectiveness of TFESI and PA in patients with LFSS. The PA group showed significantly better therapeutic outcomes as measured by VNS and ODI at the 3-month follow-up.

In two retrospective studies evaluating the effectiveness of PA, poor outcomes were more common in patients with foraminal stenosis [26,27]. Poor outcomes were attributable to the association of LSS with irreversible changes such as epidural scars and hypertrophy of bony structures and ligaments; such changes may render the nerve root refractory to management by the local application of corticosteroids $[28,29]$. Such changes may also interfere with advancement of a catheter or injection of medication into the ventral epidural space and thus contribute to relatively poor PA outcomes [26]. Unlike in the studies mentioned above, our study showed a very successful therapeutic rate ( $70 \%$ success) in foraminal stenosis patients 3 months after the procedure. It should be noted that, first, the degree of severity in radiologic findings of LSS is known to not be proportional to symptom severity [30,31]. Park et al. [32] demonstrated in cases of clinical LSS there was no association between PA outcome and anatomical degree of stenosis. In addition, Choi et al. [33] reported that LSS location was not a significant factor in the prognosis of PA. Secondly, the absence of previous lumbar surgery and root compression were identified to be good prognostic predictors of the results of PA [33]. Our study may have shown favorable outcomes since we included patients with root compression confirmed by radiologic examinations and excluded patients who had recently undergone surgery. Third, several studies have sought to determine whether epidural administration of hyaluronidase or hypertonic saline improves outcomes [22,34-36]. Yousef et al. [34] compared treatment outcomes in 38 subjects who received either FL-guided caudal injections of $10 \mathrm{~mL} 0.25 \%$ bupivacaine $+30 \mathrm{~mL} 3 \%$ hypertonic saline $+80 \mathrm{mg}$ methylprednisolone, or the same mixture with 1,500 units of hyaluronidase added. In this small prospective study, only those patients who received hyaluronidase continued to experience benefits at 6 and 12 months post-procedure [34]. Two randomized studies conducted by the same group of investigators in patients with pain from failed back surgeries or sciatica compared high-volume interlaminar epidural injections of $5 \mathrm{~mL} 0.25 \%$ bupivacaine and $80 \mathrm{mg}$ of triamcinolone, $5 \mathrm{~mL} 0.25 \%$ bupivacaine and 1,500 units of hyaluronidase, or a combination of bupivacaine, steroids and hyaluronidase $[35,36]$. In both studies, greater improvement was noted in the group that received hyaluronidase and steroids than in those who received either drug alone. Thus, there is moderate evidence supporting the use of hypertonic saline in PA, and some evidence in favor of using hyaluronidase. Our study used hyaluronidase and hypertonic saline as well, which seems to have contributed to positive outcomes. 
Fourth, a significant portion of the benefit of PA can be attributed to high injection volume. In a systemic review, Rabinovitch et al. [23] found a strong correlation between the volume of fluid injected and pain relief irrespective of steroid dose in the immediate and intermediate term and a trend towards significance in the short term.

As is the case with any procedural intervention, bleeding, infection, and nerve damage are some of the general complications associated with PA. Previous, larger studies examined complications more extensively than we did. One retrospective review revealed a variety of different complications such as bent needle tips (4.8\%), torn catheters during withdrawal $(1.2 \%)$, sheared catheter remnants $(0.4 \%)$, intrathecal placement of catheters (4.4\%), and epidural abscesses (1.2\%) [37]. In another large study, a prospective evaluation of 10,000 epidural injections found that of 839 patients who underwent adhesiolysis, the rates of intravascular injection (11.6\%), transient nerve irritation (1.9\%), and dural puncture (1.8\%) were significantly higher than in patients who underwent conventional ESI [38]. No such fatal complication was reported in this study, and only transient nerve irritation was found during both TFESI $(n=4)$ and PA $(\mathrm{n}=3)$.

The present study has several limitations. First, this study was retrospective in design. Although we selected subjects using valid inclusion and exclusion criteria, it is important to follow up our study with one that includes a more heterogeneous group of subjects. In addition, we could not evaluate the benefit of other treatments such as medication and physical therapy during the followup period. It is expected, however, that these treatments had no or minimal effects on the results because patients refractory to these treatments were chosen for this study. Second, the population was small. Even so, this study can be regarded as a significant first step that should lead to further inquiry on the effectiveness of TFESI and PA among foraminal stenosis patients. Third, a 3-month follow-up period is short. However, because the procedure was not repeated during the follow-up period, our results reflect the clinical efficacy of a single treatment and exclude the influences of repetition and the cumulative effects of multiple procedures. Fourth, the injected materials are different between the two groups. The TFESI group was injected with $0.5 \%$ lidocaine $1.5 \mathrm{~mL}+$ triamcinolone $20 \mathrm{mg}$, while the PA group was injected with $10 \mathrm{~mL} 0.25 \%$ bupivacaine $+3 \%$ hypertonic saline. Since there are differences in drug type as well as injection amount between the groups, this could be considered a method bias. Fifth, chronic lumbar foraminal stenosis is commonly accompanied by epidural adhesion. However, lumbar foraminal stenosis without adhesion is also found. Adhesions in the PA group were confirmed by filling defects, but no such assessment was done in the TFESI group. So if main pathologic condition is adhesion, we conclude that PA is the better treatment option, but if not, we cannot conclude that PA is more effective than TFESI in the treatment of chronic lumbar foraminal stenosis.

Our study suggests that PA is effective for pain reduction and functional improvement in patients with chronic radicular pain caused by LFSS. Therefore, PA should be considered for patients with previous ineffective responses to conservative treatment. Although PA seems to be more effective than TFEFI according to the results of our study, in order to make a more meaningful conclusion, a prospective study with a larger sample size will be necessary.

\section{CONFLICT OF INTEREST}

No potential conflict of interest relevant to this article was reported.

\section{REFERENCES}

1. Jenis LG, An HS. Spine update: lumbar foraminal stenosis. Spine (Phila Pa 1976) 2000;25:389-94.

2. Kunogi J, Hasue M. Diagnosis and operative treatment of intraforaminal and extraforaminal nerve root compression. Spine (Phila Pa 1976) 1991;16:1312-20.

3. Porter RW, Hibbert C, Evans C. The natural history of root entrapment syndrome. Spine (Phila Pa 1976) 1984;9:418-21.

4. Jinkins JR, Whittemore AR, Bradley WG. The anatomic basis of vertebrogenic pain and the autonomic syndrome associated with lumbar disk extrusion. AJR Am J Roentgenol 1989;152:1277-89.

5. Thomas E, Cyteval C, Abiad L, Picot MC, Taourel P, Blotman F. Efficacy of transforaminal versus interspinous corticosteroid injectionin discal radiculalgia: a prospective, randomised, double-blind study. Clin 
Rheumatol 2003;22:299-304.

6. Manchikanti L. Transforaminal lumbar epidural steroid injections. Pain Physician 2000;3:374-98.

7. Manchikanti L, Buenaventura RM, Manchikanti KN, Ruan X, Gupta S, Smith HS, et al. Effectiveness of therapeutic lumbar transforaminal epidural steroid injections in managing lumbar spinal pain. Pain Physician 2012;15:E199-245.

8. Kim SH, Choi WJ, Suh JH, Jeon SR, Hwang CJ, Koh WU, et al. Effects of transforaminal balloon treatment in patients with lumbar foraminal stenosis: a randomized, controlled, double-blind trial. Pain Physician 2013;16:213-24.

9. Fukusaki M, Kobayashi I, Hara T, Sumikawa K. Symptoms of spinal stenosis do not improve after epidural steroid injection. Clin J Pain 1998;14:148-51.

10. Smith CC, Booker T, Schaufele MK, Weiss P. Interlaminar versus transforaminal epidural steroid injections for the treatment of symptomatic lumbar spinal stenosis. Pain Med 2010;11:1511-5.

11. Lee JH, Lee SH. Clinical effectiveness of percutaneous adhesiolysis versus transforaminal epidural steroid injection in patients with postlumbar surgery syndrome. Reg Anesth Pain Med 2014;39:214-8.

12. Chopra P, Smith HS, Deer TR, Bowman RC. Role of adhesiolysis in the management of chronic spinal pain: a systematic review of effectiveness and complications. Pain Physician 2005;8:87-100.

13. Trescot AM, Chopra P, Abdi S, Datta S, Schultz DM. Systematic review of effectiveness and complications of adhesiolysis in the management of chronic spinal pain: an update. Pain Physician 2007;10:129-46.

14. Bogduk N. On the definitions and physiology of back pain, referred pain, and radicular pain. Pain 2009; 147:17-9.

15. Hartrick CT, Kovan JP, Shapiro S. The numeric rating scale for clinical pain measurement: a ratio measure? Pain Pract 2003;3:310-6.

16. Fairbank JC, Pynsent PB. The Oswestry Disability Index. Spine (Phila Pa 1976) 2000;25:2940-52.

17. Manchikanti L, Singh V, Falco FJ, Cash KA, Pampati V. Lumbar facet joint nerve blocks in managing chronic facet joint pain: one-year follow-up of a randomized, double-blind controlled trial: Clinical Trial NCT00355914. Pain Physician 2008;11:121-32.

18. Lee JH, An JH, Lee SH. Comparison of the effective- ness of interlaminar and bilateral transforaminal epidural steroid injections in treatment of patients with lumbosacral disc herniation and spinal stenosis. Clin J Pain 2009;25:206-10.

19. Kobayashi S, Takeno K, Miyazaki T, Kubota M, Shimada S, Yayama T, et al. Effects of arterial ischemia and venous congestion on the lumbar nerve root in dogs. J Orthop Res 2008;26:1533-40.

20. Cooper RG, Freemont AJ, Hoyland JA, Jenkins JP, West CG, Illingworth KJ, et al. Herniated intervertebral disc-associated periradicular fibrosis and vascular abnormalities occur without inflammatory cell infiltration. Spine (Phila Pa 1976) 1995;20:591-8.

21. Manchikanti L, Boswell MV, Rivera JJ, Pampati VS, Damron KS, McManus CD, et al. A randomized, controlled trial of spinal endoscopic adhesiolysis in chronic refractory low back and lower extremity pain [ISRCTN 16558617]. BMC Anesthesiol 2005;5:10.

22. Lee F, Jamison DE, Hurley RW, Cohen SP. Epidural lysis of adhesions. Korean J Pain 2014;27:3-15.

23. Rabinovitch DL, Peliowski A, Furlan AD. Influence of lumbar epidural injection volume on pain relief for radicular leg pain and/or low back pain. Spine J 2009;9:509-17.

24. Manchikanti L, Cash KA, McManus CD, Pampati V, Singh V, Benyamin R. The preliminary results of a comparative effectiveness evaluation of adhesiolysis and caudal epidural injections in managing chronic low back pain secondary to spinal stenosis: a randomized, equivalence controlled trial. Pain Physician 2009;12:E341-54.

25. Manchikanti L, Pampati V, Fellows B, Rivera JJ, Damron KS, Beyer C, et al. Effectiveness of percutaneous adhesiolysis with hypertonic saline neurolysis in refractory spinal stenosis. Pain Physician 2001;4:366-73.

26. Lee JH, Lee SH. Clinical effectiveness of percutaneous adhesiolysis and predictive factors of treatment efficacy in patients with lumbosacral spinal stenosis. Pain Med 2013;14:1497-504.

27. Lee JH, Lee SH. Clinical effectiveness of percutaneous adhesiolysis using Navicath for the management of chronic pain due to lumbosacral disc herniation. Pain Physician 2012;15:213-21.

28. Rivest C, Katz JN, Ferrante FM, Jamison RN. Effects of epidural steroid injection on pain due to lumbar spinal stenosis or herniated disks: a prospective study. 
Arthritis Care Res 1998;11:291-7.

29. Epter RS, Helm S 2nd, Hayek SM, Benyamin RM, Smith HS, Abdi S. Systematic review of percutaneous adhesiolysis and management of chronic low back pain in post lumbar surgery syndrome. Pain Physician 2009;12:361-78.

30. Amundsen T, Weber H, Lilleas F, Nordal HJ, Abdelnoor M, Magnaes B. Lumbar spinal stenosis. Clinical and radiologic features. Spine (Phila Pa 1976) 1995;20:1178-86.

31. Boden SD, McCowin PR, Davis DO, Dina TS, Mark AS, Wiesel S. Abnormal magnetic-resonance scans of the cervical spine in asymptomatic subjects: a prospective investigation. J Bone Joint Surg Am 1990;72:117884.

32. Park CH, Lee SH, Jung JY. Dural sac cross-sectional area does not correlate with efficacy of percutaneous adhesiolysis in single level lumbar spinal stenosis. Pain Physician 2011;14:377-82.

33. Choi E, Nahm FS, Lee PB. Evaluation of prognostic predictors of percutaneous adhesiolysis using a Racz catheter for post lumbar surgery syndrome or spinal stenosis. Pain Physician 2013;16:E531-6.

34. Yousef AA, EL-Deen AS, Al-Deeb AE. The role of adding hyaluronidase to fluoroscopically guided caudal steroid and hypertonic saline injection in patients with failed back surgery syndrome: a prospective, double-blinded, randomized study. Pain Pract 2010;10:548-53.

35. Kim SB, Lee KW, Lee JH, Kim MA, An BW. The effect of hyaluronidase in interlaminar lumbar epidural injection for failed back surgery syndrome. Ann Rehabil Med 2012;36:466-73.

36. Kim SB, Lee KW, Lee JH, Kim MA, Kim BH. The additional effect of hyaluronidase in lumbar interlaminar epidural injection. Ann Rehabil Med 2011;35:405-11.

37. Manchikanti L, Malla Y, Wargo BW, Cash KA, Pampati V, Fellows B. A prospective evaluation of complications of 10,000 fluoroscopically directed epidural injections. Pain Physician 2012;15:131-40.

38. Talu GK, Erdine S. Complications of epidural neuroplasty: a retrospective evaluation. Neuromodulation 2003;6:237-47. 